\title{
Management of Phyllodes Breast Tumors: A Case Report and Review of the Literature
}

\author{
Pinelopi Theopisti Memtsa ${ }^{\mathrm{a}, \mathrm{c}}$, Aikaterini Papadopoulou ${ }^{\mathrm{a}}$, Ariadni Kyriakogiannaki ${ }^{\mathrm{b}}$, \\ Ioannis Tzitzikas ${ }^{\mathrm{a}}$
}

\begin{abstract}
Malignant phyllodes tumor is a rare lesion of the breast that can mimic benign masses such as fibroadenomas on clinical diagnosis but is characterized by a typical rapid growth. They usually occur in middle-aged women ranging in age from 35 to 55 years old. Clinically, phyllodes tumors tend to present as enlarging pain-less breast masses that stretch the overlying skin. Treatment can be either wide local excision or mastectomy provided histologically clear specimen margins are ensured. The role of adjuvant radiotherapy and chemotherapy remains uncertain, but some results suggest that consideration should be given for their use in cases of malignant phyllodes tumors.
\end{abstract}

Keywords: Phyllodes; Radiation therapy; Breast cancer

\section{Introduction}

Phyllodes tumors are rare fibroepithelial lesions. They make up 0.3 to $0.9 \%$ of female breast tumors. They occur in women aged 35 to 55 years. The tumor is rarely found in adolescents and the elderly. They have been described as a giant type of fibroadenoma. The World Health Organization (WHO) adopted the term phyllodes tumor and as described by Rosen subclassified them histologically as benign, borderline, or malignant according to the features such as tumor margins, stromal overgrowth, tumor necrosis, cellular atypia, and number of mitosis per high power field [1-2]. The majority of phyllodes tumors have been described as benign ( $35 \%$ to $64 \%$ ), with the remainder divided between the borderline and malignant subtypes. The term phyllodes tumor represents a broad range of fibroepithelial diseases and presence of an epithelial component with stromal components differentiates the phyllodes tumor from

Manuscript submitted May 26, 2018, accepted June 20, 2018

aDepartment of Radiation Oncology, University Hospital of Thessaloniki AHEPA, Thessaloniki, Greece

bDepartment of Radiation Oncology, General Hospital of Athens ALEXANDRA, Athina, Greece

${ }^{\mathrm{c} C o r r e s p o n d i n g ~ A u t h o r: ~ P i n e l o p i ~ T h e o p i s t i ~ M e m t s a, ~ D e p a r t m e n t ~ o f ~ R a d i a t i o n ~}$ Oncology, University Hospital of Thessaloniki AHEPA, Thessaloniki, Greece. Email: pennymemtsa@hotmail.com

doi: https://doi.org/10.14740/jmc3099w other stromal sarcomas. Unlike carcinoma breast, phyllodes tumors start outside of the ducts and lobules, in the breast's connective tissue. In addition to stromal cells, phyllodes tumors can also contain cells from the ducts and lobules [2].

Accurate preoperative pathological diagnosis allows correct surgical planning and avoidance of reoperation. Malignant phyllodes tumors, if inadequately treated, have a propensity for rapid growth and metastatic spread. In contrast, benign phyllodes tumors on clinical, radiological, and cytological examination are often indistinguishable from fibroadenomas and can be cured by local surgery. With the non-operative management of fibroadenomas widely adopted, the importance of phyllodes tumors today lies in the need to differentiate them from other benign breast lesions. Treatment can be either wide local excision or mastectomy provided histologically clear specimen margins are ensured. The role of adjuvant radiotherapy and chemotherapy remains uncertain, but encouraging results using radiotherapy and chemotherapy for soft-tissue sarcomas suggest that consideration should be given for their use in cases of malignant phyllodes tumors [3].

\section{Case Report}

A 42-year-old female patient presented in our department with a 4-month history of palpable mass on the left breast. There was no family history of breast cancer. In clinical examination there was a protruding and hardened palpable mass occupying the upper outer quadrant of the left breast. Signs of axillary involvement were not evidenced.

During the diagnostic investigations, the patient was submitted to mammography and ultrasound. Mammogram showed a hyper dense rounded mass measuring approximately $30 \mathrm{~mm}$ with well-defined margins in the left breast. Ultrasound showed rounded confluent lesion with well-defined and regular contour, with cystic areas in its interior, posterior acoustic enhancement and internal flow to color-flow Doppler examination.

Ultrasound-guided percutaneous biopsy (core biopsy) was performed on the breast lesion, which revealed a biphasic neoplasm with malignant stromal component.

Because of the malignant result on core biopsy the patient underwent left wide local excision with sentinel lymph node biopsy. Surgical margins were free of tumor and axilla was clear. The histopathological analysis of the surgical specimen confirmed the diagnosis of a malignant phyllodes tumor. 
The patient underwent adjuvant radiotherapy in the left breast and received 40 Gy in 15 fractions with IMRT technique. No radiation related skin toxicity was seen. The patient continues being followed up in a yearly basis.

\section{Discussion}

Malignant phyllodes tumor is a rare lesion of the breast that can mimic benign masses such as fibroadenomas on clinical diagnosis but is characterized by a typical rapid growth. They usually occur in middle-aged women ranging in age from 35 to 55 years old. They are classified into benign, malignant, and borderline tumor according to histopathologic features. They are rare fibroepithelial neoplasms, which represent roughly $0.3 \%-0.9 \%$ of all breast cancers. Like fibroadenomas, phyllodes tumors are composed of epithelial elements and a connective tissue stroma, but phyllodes tumors have higher stromal cellularity [4].

The term "phyllodes" means leaf like and refers to the papillary projections that are seen on microscopic examination. The diagnosis of phyllodes has been well established in commendations of the WHO [1]. A benign phyllodes tumor is characterized by mildly increased stromal cellularity and irregular borders. In contrast, a fibroadenoma whilst may have increased stromal cellularity usually has a circumscribed margin but this cannot be assessed on the biopsy. Therefore it may be difficult to distinguish a fibroadenoma and phyllodes tumor on biopsy. Furthermore, the distinction of benign and borderline phyllodes using the criteria of increased stromal cellularity, stromal atypia and mitoses can be very subjective and hence the accurate diagnosis is usually made only on the excision specimen. A malignant phyllodes tumor is distinguished from a benign/borderline phyllodes tumor by the presence of marked stromal cellularity, cellular atypia and mitotic activity of at least 10/10 high power field [5].

Surgical resection remains the gold standard of treatment, whereas radiation therapy and chemotherapy have a more undefined role. Most studies recommend a more than 1- to 2-cm excision margin based on the evidence that local recurrence occurs more frequently in patients with narrow surgical margins less than $1-2 \mathrm{~cm}$. However, an excision of this size is not possible because of the large size of the tumor and the minimal remaining normal breast tissue.

Clinically, phyllodes tumors tend to present as enlarging pain-less breast masses that stretch the overlying skin. Though ulceration and nipple retraction have been reported, they remain uncommon. The median size of phyllodes tumors are usually $4 \mathrm{~cm}$ though recently in the literature there has been reports of large tumor size up to $50 \mathrm{~cm}$ and these are mostly malignant. Palpable axillary lymphadenopathy has been reported in up to $20 \%$ of cases but these are often reactive in nature and metastatic involvement of axillary lymph nodes is extremely rare [6].

There are no pathognomonic mammographic or ultrasound features of phyllodes tumor. However, a recent retrospective study suggests a tumor size of greater than $3 \mathrm{~cm}$, irregular shape, micro lobulated margins, complex internal echo pattern and hyper vascularity were significant findings of phyllodes tumor. Presence of internal cystic areas on MRI also was predictive of phyllodes tumors. Thus imaging may still serve as an aid to identify phyllodes tumor [7]. However, a core biopsy remains the best investigation for preoperative diagnosis. Though most cellular fibroepithelial lesions are fibroadenomas, surgeons should take into account the clinical presentation, image findings and the pathologist input how likely a phyllodes is present. This allows planning of the intended surgical margins [8].

Surgical management is the mainstay but the type of surgery has been a source of debate over the years. Studies have shown no differences between breast conserving surgery versus mastectomy in terms of metastasis-free survival or overall survival, despite the higher incidence of local recurrence that comes with breast conserving surgery. If diagnosed preoperatively, tumor should be resected with at least $1 \mathrm{~cm}$ margins particularly in the borderline and malignant phyllodes tumors. This can be accomplished by either lumpectomy or mastectomy, depending upon the size of the tumor relative to the breast. Local recurrence and 5-year survival rates of $4 \%$ and $96 \%$ respectively have been reported for benign phyllodes tumors diagnosed after local excision of what appeared to be a fibroadenoma [9]. Whether patients with benign phyllodes tumors who have undergone local excision and have histologically positive specimen margins should undergo further surgery or be entered in surveillance program remains controversial. Re-excision of borderline and malignant phyllodes tumors identified after local excision should be considered. Successful management of phyllodes tumor involves wide excision with adequate margins. Most advocate a surgical margin of $1 \mathrm{~cm}$. Routine axillary dissection is not recommended [10].

Adjuvant chemotherapy and radiotherapy have not been proven to be useful in the treatment of phyllodes tumor. Adjuvant radiotherapy has been proven to be beneficial in patients with adverse features like bulky tumors, close or positive surgical margins, hyper cellular stroma, high nuclear pleomorphism, high mitotic rate, presence of necrosis, and increased vascularity within the tumor and tumor recurrence. Radiotherapy is usually recommended only for cases with positive or near-positive surgical margins and selected cases whose further surgical procedures cannot be performed [11]. Chemotherapy, including anthracyclines, cisplatin, and etoposide, has been mentioned in various studies but with no survival advantage. The use of hormonal therapy, such as tamoxifen, has not been fully investigated in cystosarcoma phyllodes. Still, the use of endocrine therapy in either the adjuvant or palliative setting has not been extensively studied [12].

The prognosis for malignant phyllodes tumors is poor, and the role of the variety of treatment modalities is not clearly defined because of how rare the disease actually is. The most common path of spread is hematogenous, which occurs mostly in the lungs, pleura, and bones, such as in sarcoma [9].

\section{Conclusions}

Phyllodes tumors are rare causes of rapidly enlarging painless breast masses in middle age women. A core biopsy is needed 
for diagnosis but often, definitive diagnosis is only obtained after excision. The surgeon should take into account the clinical and imaging characteristics to assess the likelihood of presence of phyllodes to decide the extent of margins needed during surgery. The patient must be adequately counselled for possibility of further surgery and adjuvant treatment depending on the final histology. Further studies should be done so as to establish a golden standard treatment for the overall management of this rare entity.

\section{References}

1. Lakhani SR, Ellis IO, Schnitt SJ, et al. WHO classification of tumours of the breast. (ed). International Agency for Research on Cancer, World Health Organization, Lyon; 2012.

2. Tan PH, Jayabaskar T, Chuah KL, Lee HY, Tan Y, Hilmy M, Hung H, et al. Phyllodes tumors of the breast: the role of pathologic parameters. Am J Clin Pathol. 2005;123(4):529-540.

3. Sin EI, Wong CY, Yong WS, Ong KW, Madhukumar P, Tan VK, Thike AA, et al. Breast carcinoma and phyllodes tumour: a case series. J Clin Pathol. 2016;69(4):364-369.

4. Barrio AV, Clark BD, Goldberg JI, Hoque LW, Bernik SF, Flynn LW, Susnik B, et al. Clinicopathologic features and long-term outcomes of 293 phyllodes tumors of the breast. Ann Surg Oncol. 2007;14(10):2961-2970.
5. Sawyer EJ, Hanby AM, Ellis P, Lakhani SR, Ellis IO, Boyle S, Tomlinson IP. Molecular analysis of phyllodes tumors reveals distinct changes in the epithelial and stromal components. Am J Pathol. 2000;156(3):1093-1098.

6. Tan BY, Acs G, Apple SK, Badve S, Bleiweiss IJ, et al. Phyllodes tumours of the breast a concensus review. Histopathology. 2016;(6891):5-21.

7. Testori A, Meroni S, Errico V, Travaglini R, Voulaz E, Alloisio M. Huge malignant phyllodes breast tumor: a real entity in a new era of early breast cancer. World J Surg Oncol. 2015;13:81.

8. Roberts N, Runk DM. Aggressive malignant phyllodes tumor. Int J Surg Case Rep. 2015;8C:161-165.

9. Reinfuss M, Mitus J, Duda K, Stelmach A, Rys J, Smolak $\mathrm{K}$. The treatment and prognosis of patients with phyllodes tumor of the breast: an analysis of 170 cases. Cancer. 1996;77(5):910-916.

10. Ramakant P, Chakravarthy S, Cherian JA, Abraham DT, Paul MJ. Challenges in management of phyllodes tumors of the breast: a retrospective analysis of 150 patients. Indian J Cancer. 2013;50(4):345-348.

11. Chaney AW, Pollack A, McNeese MD, Zagars GK. Adjuvant radiotherapy for phyllodes tumor of breast. Radiat Oncol Investig. 1998;6(6):264-267.

12. Burton GV, Hart LL, Leight GS, Jr., Iglehart JD, McCarty KS, Jr., Cox EB. Cystosarcoma phyllodes. Effective therapy with cisplatin and etoposide chemotherapy. Cancer. 1989;63(11):2088-2092. 